\title{
An Introduction to Trialing Intrathecal Baclofen in Patients with Hemiparetic Spasticity: A Description of 3 Cases
}

Michael E. Harned, MD, Sara S. Salles, DO and Jay S. Grider, DO, PhD

From: University of Kentucky, Lexington, $\mathrm{KY}$.

Dr. Harned is Assistant Professor, Department of Anesthesiology, Division of Pain Medicine, University of Kentucky, Lexington,

$\mathrm{KY}$.

Dr. Salles is Associate Professor, Department of Physical Medicine \& Rehabilitation, University of Kentucky, Lexington, KY.

Dr. Grider is Associate Professor,

Department of Anesthesiology, Division of Pain Medicine, University of Kentucky, Lexington,

KY.

Address correspondence: Michael E. Harned, MD Assistant Professor University of Kentucky College of Medicine

800 Rose Street Suite N 201 Lexington, KY 40536

E-mail:meharn2@email.uky.edu

Disclaimer: There was no external funding in the preparation of this manuscript. Conflict of interest: None.

Manuscript received: $03 / 11 / 2011$ Revised manuscript received: 07/22/2011 Accepted for publication: 08/01/2011

Free full manuscript: www.painphysicianjournal.com
Background: Intrathecal baclofen has been an effective therapy in the management of spasticity. As interventional pain physicians are rapidly becoming the experts in intrathecal drug delivery, they are now frequently asked to trial and implant intrathecal baclofen therapy. While some physicians might be very comfortable with the process of trialing and implanting, others will have next to no experience until the first consult appears on their desks. While uncomplicated lower extremity spasticity can usually be trialed with a single-shot bolus injection, more complicated cases of upper and lower extremities or hemiparetic spasticity need a more delicate approach. This is the first case series in the literature reporting a trial using an indwelling temporary catheter and inpatient admission. Moreover, while the technical aspects of intrathecal therapy trialing and implantation might be familiar for the interventional physician, we review the indications and goals of therapy, about which the physician may be less familiar.

Objective: To present a technique for trialing intrathecal baclofen in patients with severe upper and lower extremities spasticity complication for which a single shot technique may be inadequate.

Design: Case report of three patients.

Setting: Tertiary-care referral medical center.

Patients: A 30-year-old man with severe spasticity of the right upper and lower extremities with preserved left-sided function due to diffuse axonal injury. A 45-year-old woman with traumatic brain injury and severe spasticity of the left upper extremity with minimal dysfunction in the right upper extremity. A 34-year-old woman with Multiple Sclerosis and severe spasticity in the right upper extremity and bilateral lower extremities.

Intervention: Placement of a temporary intrathecal catheter and an inpatient trial of intrathecal baclofen.

Results: In all patients, there was significant improvement in spasticity as documented by decreased Modified Ashworth Scale scores while preserving motor strength and coordination in the unaffected extremities.

Limitations: Retrospective review of 3 cases in a single center.

Conclusions: Trialing for baclofen is usually performed as a single shot bolus. For patients with severe hemiparetic spasticity or in patients where weakness in the unaffected limb might significantly affect quality of life, this trialing technique may be inadequate. In these patients, placement of a temporary intrathecal catheter and inpatient admission may be a more effective trial method.

Key words: Baclofen, intrathecal drug delivery, spasticity, multiple sclerosis, traumatic brain injury

Pain Physician 2011; 14: 483-489 
S ince it was approved for use in 1984, intrathecal baclofen has proven effective in the management of severe spasticity resistant to oral medications (1-4). Baclofen is a structural analog of the inhibitory neurotransmitter gamma-aminobutyric acid (GABA), which is used as an agent in the management of spasticity (1). Spasticity can be characterized by its positive or negative symptoms. The positive symptoms are often velocity-dependent hypertonicity, hyperreflexia and clonus. Negative symptoms tend to be that of weakness, dyscoordination, pain, and limited functional abilities (5). While the precise mechanism of action is not fully understood, baclofen does inhibit monosynaptic and polysynaptic reflexes at the spinal level (6). It is felt that this results in decreased excitatory neurotransmitter release at terminals of primary afferents (6). As an oral agent, baclofen is used in the management of spasticity and spasms secondary to cerebral palsy, multiple sclerosis, and brain or spinal cord injury. In cases of severe spasticity, symptoms can be difficult to control with oral medications. Moreover, some patients suffer intolerable central nervous side effects such as sedation or somnolence with oral administration (7). In these cases, intrathecal baclofen (ITB) delivered via a spinal catheter and an implanted pump has proven beneficial $(1-3,8,9)$.

Prior to implantation of an intrathecal drug delivery device (IDDS), a trial is performed to document that ITB is an effective therapy for managing symptoms. A trial usually consists of a single bolus injection of between 50 and $100 \mu \mathrm{g}$ of baclofen into the intrathecal space $(10,11)$. The patient is then observed in the practitioner's office for improvement in Modified Ashworth Scale (MAS), the most commonly accepted rating scale for spasticity (Table 1) (11-13). However, patients may have both upper and lower extremities symptoms; improvement in upper extremity symptoms is difficult to discern from a low lumbar, single-shot technique. Moreover, patients may have asymmetric disease, where an upper extremity may have severe rigidity and spasticity while the contralateral limb has well-preserved strength and no spasticity. The singleshot technique may provide good relaxation of the affected extremity but undesirable weakness in the nonaffected extremity. Therefore, a single shot trial may be a poor technique in helping patients decide if ITB will provided the desired outcomes. We present our protocol utilizing an indwelling intrathecal trial catheter, inpatient admission and twice-daily physical therapy assessments to document symptom improvement. We have found that this trialing process helps both the patient and physician make a more informed decision regarding ITB for severe spasticity.

\section{Methods}

\section{Intrathecal Baclofen Protocol}

At the University of Kentucky, Lexington, $K Y$, patients with either spastic hemiparesis or a spastic upper extremity with a preserved contralateral extremity are considered candidates for an ITB catheter trial. On the morning of the trial, all antispasmotic medicines are held and a pre-catheter placement examination is completed by the physical therapist, documenting the MAS at each muscle group in the upper and lower extremities bilaterally (Table 2). Next, under strict aseptic conditions, a temporary intrathecal catheter is placed via a low lumbar paramedian approach using local anesthesia and no more than $2 \mathrm{mg}$ of midazolam intravenously. The catheter is advanced to the T1 vertebral level using direct fluoroscopic guidance and then tunneled subcutaneously to the lateral flank. Patients are transferred to a designated inpatient ward familiar with the ITB clinical protocol. An initial intrathecal bolus of $50 \mu \mathrm{g}$ of baclofen is then administered and the patient is evaluated at 2 and 4 hours postbolus. The primary end point

Table 1. Modified Ashworth Scale.

\begin{tabular}{|c|l|}
\hline Score & \multicolumn{1}{|c|}{ Criteria } \\
\hline 0 & No increase in tone \\
\hline 1 & $\begin{array}{l}\text { Slight increase in muscle tone, manifested by a catch and release or by minimal resistance at the end of the range of motion } \\
\text { when the affected part (s) is moved in flexion or extension. }\end{array}$ \\
\hline $1+$ & $\begin{array}{l}\text { Slight increase in muscle tone, manifested by a catch, followed by minimal restistance throughout the remainder (less than half) } \\
\text { of the range of motion. }\end{array}$ \\
\hline 2 & More marked increase in muscle tone through most of the range of motion, but afected part (s) easily moved \\
\hline 3 & Considerable increase in muscle tone; passive movement difficult \\
\hline 4 & Affected part(s) rigid in flexion or extension \\
\hline
\end{tabular}


Table 2. Intrathecal baclofen catheter trial assessment.

\begin{tabular}{|c|c|c|c|c|c|c|c|c|c|c|c|}
\hline \multirow{2}{*}{\multicolumn{2}{|c|}{\begin{tabular}{|c|} 
Spasticity \\
$(1-5$ to see reverse $)$
\end{tabular}}} & \multicolumn{2}{|c|}{$\begin{array}{l}\text { Pre-Bolus } \\
\text { (baseline) }\end{array}$} & \multicolumn{2}{|c|}{$\begin{array}{l}2 \text { Hour Post- } \\
\text { Bolus }\end{array}$} & \multicolumn{2}{|c|}{$\begin{array}{l}\text { 4-Hour Post- } \\
\text { Bolus }\end{array}$} & \multicolumn{2}{|c|}{$\begin{array}{c}\text { 6-Hour Post- } \\
\text { Bolus }\end{array}$} & \multicolumn{2}{|c|}{$\begin{array}{c}\text { 8-Hour Post } \\
\text { Bolus }\end{array}$} \\
\hline & & $\mathbf{L}$ & $\mathbf{R}$ & $\mathbf{L}$ & $\mathbf{R}$ & $\mathbf{L}$ & $\mathbf{R}$ & $\mathbf{L}$ & $\mathbf{R}$ & $\mathbf{L}$ & $\mathbf{R}$ \\
\hline \multirow{6}{*}{ 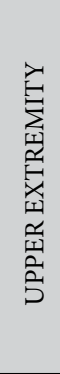 } & Wrist Flexion & & & & & & & & & & \\
\hline & Wrist Extension & & & & & & & & & & \\
\hline & Elbow Flexion & & & & & & & & & & \\
\hline & Other: & & & & & & & & & & \\
\hline & Other & & & & & & & & & & \\
\hline & $\begin{array}{l}\text { Average Upper } \\
\text { Extremity Score }\end{array}$ & & & & & & & & & & \\
\hline \multirow{10}{*}{ 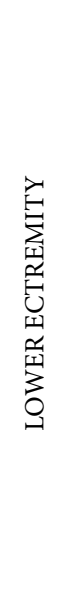 } & Hip Abduction & & & & & & & & & & \\
\hline & Hip Abduction & & & & & & & & & & \\
\hline & Hip Extension & & & & & & & & & & \\
\hline & Knee Extension & & & & & & & & & & \\
\hline & Knee Flexion & & & & & & & & & & \\
\hline & Ankle Dorsiflexion & & & & & & & & & & \\
\hline & Plantar Flexion & & & & & & & & & & \\
\hline & Other & & & & & & & & & & \\
\hline & Other & & & & & & & & & & \\
\hline & $\begin{array}{l}\text { Average Lower } \\
\text { Extremity Score }\end{array}$ & & & & & & & & & & \\
\hline \multicolumn{2}{|c|}{$\begin{array}{l}\text { Overall Spasm Score } \\
\text { ( } 0 \text { to } 4 \text { - see reverse) }\end{array}$} & & & & & & & & & & \\
\hline \multicolumn{2}{|c|}{$\begin{array}{l}\text { Overall Pain Score } \\
\text { (0 to } 10 \text { - see reverse) }\end{array}$} & & & & & & & & & & \\
\hline
\end{tabular}

Pre-Bolus Baseline Assessment (Subjective): Patient's tone interferes with and/or impacts: (circle all that apply) Bed Mobility Bed Positioning Passive ROM Active ROM ADL Skin Integrity Sitting Position Standing Alignment Others: Sleeping Transfers Gait Balance Pain Energy Demands Caregiver Demands

is a reduction in the MAS of one for centrally mediated spasticity and a reduction of 2 points for spasticity secondary to spinal mechanisms $(10,11)$. The secondary end point is preservation of strength and function in the unaffected extremity.

After the 4-hour postbolus exam, a continuous infusion is begun to find the optimum dose for implantation. The initial dose is $100 \mu \mathrm{g} / \mathrm{d}$ and is titrated in increments of $100 \mu$ guntil primary and secondary end points are meet. Once an acceptable dose is obtained and improvement is documented, the catheter is removed and the patient is kept flat for one hour to minimize the chance of postdural puncture headache prior to discharge. Approximately 2 weeks later, the permanent system is implanted. After implantation, patients are transferred to an inpatient rehabilitation hospital for precise titration of the pump, weaning of oral medications, and extensive rehabilitation. 


\section{Results}

The following case reports demonstrate application of our intrathecal baclofen protocol.

\section{Case 1}

Case 1 is a 30 -year-old man with diffuse axonal injury and severe traumatic brain injury secondary to a motor vehicle accident. After a prolonged hospital course, including sepsis and pulmonary failure requiring tracheostomy, the patient had residual right upper and lower extremities spasticity. Moreover, the patient was developing painful contractures while having remarkably well preserved left upper and lower extremities range of motion and function. At this point, the patient's rehabilitation and nursing care were being limited by severe spasticity in addition to the progressive development of contractures. The patient was not able to tolerate titration of baclofen due to sedation and worsening cognitive function caused by the higher doses of oral baclofen. The decision was made for a trial of ITB using a temporary catheter and inpatient obser- vation to assess improvement in right-sided spasticity and preservation of left-sided function.

Preoperatively, the patient had a MAS a of 4 out of 4 in the right upper and lower extremities (Table 3). There was no spasticity in either the left upper or lower extremities. After placing the temporary catheter, the initial $50 \mu \mathrm{g}$ bolus resulted in a rapid reduction of the MAS to 3 out of 4 in the lower extremity, but no significant change in the affected upper extremity. Moreover, the 4-hour postbolus examination demonstrated a return to the baseline MAS. Consequently, an infusion was begun at $100 \mu \mathrm{g} / \mathrm{d}$ resulting in a reduction of the MAS equal to the bolus dose. On hospital day number 2, the dose was titrated up to $200 \mu \mathrm{g} / \mathrm{d}$; the patient had continued improvement in the right lower extremity, resulting in an Ashworth of 2 out of 4 . His upper extremity score improved to 3 out of 4 . Moreover, the patient retained good strength and function in his left upper and lower extremities. Therefore, the trial was stopped and the catheter was removed. The patient underwent permanent implant and was transferred to inpatient rehabilitation at the hospital.

Table 3. MAS at each dose.

\begin{tabular}{|c|c|c|c|c|}
\hline Case & Left Upper Extremity & Right Upper Extremity & Left Lower Extremity & Right Lower Extremity \\
\hline \multicolumn{5}{|l|}{ Case \# 1} \\
\hline Pre-Bolus & $\mathrm{nl}$ & 4 & $\mathrm{nl}$ & 4 \\
\hline Post Bolus & $\mathrm{nl}$ & 4 & $\mathrm{nl}$ & 3 \\
\hline $100 \mu \mathrm{g} / \mathrm{d}$ & $\mathrm{nl}$ & 4 & $\mathrm{nl}$ & 3 \\
\hline $200 \mu \mathrm{g} / \mathrm{d}$ & $\mathrm{nl}$ & 3 & $\mathrm{nl}$ & 2 \\
\hline \multicolumn{5}{|l|}{ Case \#2 } \\
\hline Pre Bolus & 3 & $\mathrm{nl}$ & dystonic & $\mathrm{nl}$ \\
\hline Post Bolus & 3 & $\mathrm{nl}$ & dystonic & $\mathrm{nl}$ \\
\hline $100 \mu \mathrm{g} / \mathrm{d}$ & 3 & $\mathrm{nl}$ & improved & $\mathrm{nl}$ \\
\hline $200 \mu \mathrm{g} / \mathrm{d}$ & 3 & $\mathrm{nl}$ & improved & $\mathrm{nl}$ \\
\hline $400 \mu \mathrm{g} / \mathrm{d}$ & 3 & $\mathrm{nl}$ & improved & $\mathrm{nl}$ \\
\hline $700 \mu \mathrm{g} / \mathrm{d}$ & 1 & $\mathrm{nl}$ & improved & $\mathrm{nl}$ \\
\hline \multicolumn{5}{|l|}{ Case \#3 } \\
\hline Pre Bolus & $\mathrm{nl}$ & 3 & 3 & 3 \\
\hline Post Bolus & $\mathrm{nl}$ & 0 & 2 & 2 \\
\hline $100 \mu \mathrm{g} / \mathrm{d}$ & $\mathrm{nl}$ & 0 & 1 & 1 \\
\hline $200 \mu \mathrm{g} / \mathrm{d}$ & $\mathrm{nl}$ & 0 & 1 & 1 \\
\hline
\end{tabular}




\section{Case 2}

Case 2 is a 45-year-old woman with a long-standing history of traumatic brain injury after being struck by a vehicle at 7 years old. The patient had significant spasticity in the left upper extremity while having minimal spasticity in the right upper extremity, which was important in maintaining her current level of function. Moreover, while the patient had little spasticity in the lower extremities, her coexisting dystonia of the left lower extremity resulted in difficulty with ambulation and activities of daily living. The patient had been managed for a long period of time on oral baclofen as well as tizanidine but they had lost efficacy in the past several years. Moreover, with increasing doses, the patient began to develop somnolence, which was a concern given her concomitant regimen of oral opioids for the management of chronic pain. Due to the lack of efficacy with oral antispasmotics, a trial of ITB was recommended. In consideration of maintaining right upper extremity strength while modifying left upper extremity spasticity, a catheter trial with inpatient evaluation was planned.

Preoperatively, the patient had an MAS of 3 out of 4 in all muscle groups of the left upper extremity (Table 1). At 2 and 4 hours postbolus, the patient had no significant improvement in her MAS, but the physical therapist did note improved gait at the 4-hour postbolus interval. Therefore, initiation of a continuous infusion at $100 \mu \mathrm{g} / \mathrm{d}$ was begun. This was titrated up to $300 \mu \mathrm{g} / \mathrm{d}$ over the course of hospital day 2. While there was minimal improvement in spasticity, the patient was having no side effects from the therapy and further titration was initiated. On hospital day 3 the ITB dose was escalated to $700 \mu \mathrm{g} / \mathrm{d}$. At this dose the patient had significant improvement in her left sided spasticity and lower extremity dystonia. Objective measurements by physical therapy noted a reduction in her MAS to 1 out of 4 in all left upper and lower extremity muscle groups. Moreover, the patient noted improvements in her speech and ease of self-care without contralateral weakness. The catheter was removed and the patient observed overnight secondary to the relatively high dose of baclofen during the trial. The patient was then discharged home on hospital day 4 with resumption of oral baclofen. The patient returned soon thereafter for implantation of a permanent system with inpatient rehabilitation following surgery.

\section{Case 3}

Case 3 is a 34-year-old woman with a history of primary progressive multiple sclerosis which had re- mained stable for a number of years without relapse. However, in the past year during a course of pregnancy with her first child, she experienced a significant and severe exacerbation of symptoms. The patient had essentially lost the use of her right hand secondary to dyscoordination, relying on her left hand for all activities of daily living as well as other activities such as typing and bookkeeping Moreover, the spasticity in her lower extremities had progressed to the point where it was difficult for her to ambulate and she was now dependent upon a rolling walker for short distance mobility and a scooter for long distance mobility. At approximately 5 months postpartum her symptoms had not resolved and appeared to be unremitting. Oral baclofen was only minimally successful in controlling symptoms and also caused significant sedation. A trial of ITB utilizing a temporary catheter and inpatient admission were planned to document improvement in her right upper and lower extremities symptoms without attempting to preserve strength in her left upper extremity.

Preoperatively, the patient's MAS were 3 out of 4 in the lower extremities and right upper extremity (Table 1). After placing an intrathecal catheter at $T 1$, the initial $50 \mu$ gbolus demonstrated significant improvement in both upper and lower extremities. Her MAS at both 2 and 4 hours postbolus were 2 out of 4 in the lower extremities and 0 out of 4 in the right upper extremity. Fortunately, the patient developed no significant weakness or loss of function in the left upper extremity.

Given that this patient had documented success on the initial bolus, we elected to continue with the trial in order to titrate to the exact dose of maximal symptom improvement without adverse effects. Therefore, a continuous infusion of baclofen was begun at 100 $\mu \mathrm{g} / \mathrm{d}$. This was titrated up to $200 \mu \mathrm{g} / \mathrm{d}$. At this point the patient was ambulating with minimal assistance; MAS improved to 1 out of 4 in the bilateral lower extremities and continued to be 0 out of 4 in the right upper extremity. Moreover, the patient continued to have good function in the left upper extremity. The patient's catheter was removed on hospital day 2 and she was discharged home. The patient followed up in 2 weeks for implantation of a permanent system and was then transferred to inpatient rehabilitation.

\section{Discussion}

Interventional pain physicians have become comfortable with the management of IDDS for chronic pain $(2,3,14-23)$. While technically similar in use, the goals of therapy are very different. Unlike the subjective evalu- 
ation of chronic pain patients, baclofen therapy yields objective improvements visible to both the patient and physician. A familiarity with IDDS and a refreshing ability to see the efficacy of therapy means the interventional pain physician is the clinician of choice to continue providing this therapy.

When oral antispasmotics including baclofen fail to provide significant improvement in spasticity, intrathecal administration has been proven beneficial $(8,24)$. While this therapy can be highly effective, ITB is not without its risks. This can include mechanical problems with failures of the pump or loss of integrity in the intrathecal catheter. Regardless of the reason, abrupt cessation of baclofen can result in life-threatening withdrawal. Finally, there remains the risk of infection with any implanted device (25-28). With these risks accounted for, the therapy has made dramatic improvements in patients' quality of life and ease of care delivered to patients $(8,24,28)$.

ITB is not a therapy to be approached lightly, and to that end requires a trial to document efficacy in controlling symptoms. This trial has routinely been performed in the office of the practitioner followed by observation documenting improvement in spasticity based upon the MAS (10). Despite some disagreement in recent publications, Ashworth scale point reductions have been used in most studies as the measurement tool of choice as a means to document improvement in spasticity (13). Currently, it is believed that a reduction in the MAS of one point for spasticity from a cerebral mechanism and a 2 point reduction in MAS for spinal cord mediated pathology are required to consider the trial a success and proceed with implantation of a permanent catheter and subcutaneous pump device $(10,11,29)$.

When patients present with spasticity that is asymmetric, a clinical dilemma arises regarding adequate relief from the spastic symptoms while preserving function in the unaffected extremity. Single bolus trials may mislead patients who perceive significant improvement in the affected extremity while overlooking the amount of weakness experienced in the unaffected limb. In all 3 cases reported here, it was paramount to document preservation of function in the uninvolved extremities. This was more easily done with the catheter trial where changes could be observed over time, rather than in a busy office setting.

Moreover, in patients with both upper and lower extremities weakness, a lumbar single shot bolus may simply not result in improvement of upper extremity spasticity. Traditionally, it was felt that baclofen was ineffective in managing upper extremity symptoms; however, this was likely due to the use of lumber catheters. In recent years, treatment of upper extremity spasticity has become more commonplace as the catheter tip position has been pushed cephalad (30). Cases \#1 and \#3 needed both upper and lower extremity symptom relief. The safest way to deposit intrathecal baclofen is via a catheter placed in the lumbar space and advanced into the upper thoracic region. Therefore, in both these cases, a temporary catheter was not only novel but also necessary.

An additional advantage to the catheter trial was noted in case \#2. This patient received no benefit from the initial $50 \mu \mathrm{g}$ bolus and it was not until a much larger dose that efficacy was achieved. In the setting of standard single shot trialing or even repeated single shots, this patient would have been missed as an ITB responder. The literature would support single shots in the dosage range of up to $100 \mu \mathrm{g}$ but as patient \#2 demonstrated, that dose would have been insufficient to document response to ITB (10).

\section{Conclusions}

Currently, a single shot intrathecal baclofen dose is used as a trial to assess the efficacy for long-term therapy. This is an accepted practice, which is reasonable in patients with lower extremity spasticity where intrathecal baclofen placed in the lumbar spine is efficacious. However, when more complex patients are being considered for permanent implantation of an IDDS, we feel that a more sophisticated approach gives both the clinician and patient better information. We have reported a series of 3 cases where a temporary catheter was used to document control of spasticity in patients with both upper and lower extremity symptoms without causing weakness in the contralateral extremity and preserved function. Moreover, we were able to identify a candidate for long term ITB who would have been missed by a single shot trial due to the need for higher doses in order to obtain efficacy.

\section{Acknowledgments}

We would like to thank the editorial board of Pain Physician for review and constructive criticism in improving the manuscript. 


\section{References}

1. Penn RD, Kroin JS. Intrathecal baclofen alleviates spinal cord spasticity [letter]. Lancet 1984; 1:1078.

2. Hayek SM, Deer TR, Pope JE, Panchal S), Patel VB. Intrathecal therapy for cancer and non-cancer pain. Pain Physician 2011; 14:219-248.

3. Patel VB, Manchikanti L, Singh V, Schultz DM, Hayek SM, Smith HS. Systematic review of intrathecal infusion systems for long-term management of chronic non-cancer pain. Pain Physician 2009; 12:345-360.

4. Deer TR, Smith HS, Burton AW, Pope JE, Doleys DM, Levy RM, Staats Peter S, Wallace MS, Webster LR, Rauck $R L$, Cousins M. Comprehensive consensus based guidelines on intrathecal drug delivery systems in the treatment of pain caused by cancer pain. Pain Physician 2011; 14: E283-E312.

5. Haley SM, Inacio CA. Evaluation of spasticity and its effect on motor function. In: Glenn MB, Whyte J(eds). The Practical Management of Spasticity in Children and Adults. Lea \& Febiger, Malvern, PA, 1990, pp 115-126.

6. Krach LE. Pharmacotherapy of spasticity: Oral medications and intrathecal baclofen. J Child Neurol 2001; 16:31-36.

7. Albright AL. Spastic cerebral palsy; approaches to drug treatment. CNS Drugs 1995; 4:17-27.

8. Albright AL, Cervi A, Singletary J. Intrathecal baclofen for spasticity in cerebral palsy. JAMA 1991; 265:1418-1422.

9. Murphy, NA, Irwin MCN, Hoff C. Intrathecal baclofen therapy in children with cerebral palsy: Efficacy and complications. Arch Phys Med Rehabil 2002; 83:1721-1725.

10. Rawicki B. Treatment of cerebral origin spasticity with continuous intrathecal baclofen delivered via an implantable pump: Long-term follow-up review of 18 patients. J Neurosurg 1999; 91:733736.

11. Awaad Y, Tayem H, Munoz S, Ham S, Michon AM, Awaad R. Functional assessment following intrathecal baclofen therapy in children with spastic cerebral palsy. J Child Neurol 2003; 18:26-34.

12. Ashworth B. Preliminary trial of cariso- prodol in multiple sclerosis. Practitioner 1964; 192:540-542.

13. Damiano DL, Quinlivan JM, Owen BF, Payne P, Nelson KC, Abel MF. What does the Ashworth Scale really measure and are instrumented measures more valid and precise? Dev Med Child Neurol 2002; 44:112-118.

14. Raffaili W, Sarti D, Demartini L, Sotgiu A, Bonezzi C, Italian Ziconotide Group. Italian Registry on long-term intrathecal ziconotide treatment. Pain Physician 2011; 14: 15-24.

15. Lima RM, Navarro LH, Carness JM, Barros GA, Margues ME, Solanki D, Ganem EM. Clinical and histological effects of the intrathecal administration of methylprednisolone in dogs. Pain Physician 2010; 13:493-501.

16. Kapoor R, Liu J, Devasenapathy A, Gordin V. Gadolinium encephalopathy after intrathecal gadolinium injection. Pain Physician 2010; 13: E321-E326.

17. Ruan X, Couch JP, Liu HN, Shah RV, Wang F, Chiravuri S. Respiratory failure following delayed intrathecal morphine pump refill: A valuable, but costly lesson. Pain Physician 2010; 13:337-341.

18. Dureja GP, Usmani H, Khan M, Tahseen $M$, Jamal A. Efficacy of intrathecal midazolam with or without epidural methylprednisolone for management of postherpetic neuralgia involving lumbosacral dermatomes. Pain Physician 2010; 13:213-221.

19. Deer TR, Smith HS, Cousins M, Doleys DM, Levy RM, Rathmell JP, Staats PS, Wallace MS, Webster LR. Consensus guidelines for the selection and implantation of patients with noncancer pain for intrathecal drug delivery. Pain Physician 2010; 13:E1750E213.

20. Wilkes D, Cook M, Solanki D. Intrathecal catheter-syringe adaptor for short-term intrathecal analgesia with an externalized pump: A case report. Pain Physician 2010; 13:151-156.

21. Burton AW, Deer TR, Wallace MS, Rauck RL, Grigsby E. Considerations and methodology for trialing ziconotide. Pain Physician 2010; 16:23-33.

22. Deer TR, Kim C, Bowman R, Tolentino D, Stewart C, Tolentino W. Intrathecal zi- conotide and opioid combination therapy for noncancer pain: An observational report. Pain Physician 2009; 12:e291E296.

23. Patel VB, Manchikanti L, Singh V, Schultz DM, Hayek SM, Smith HS. Systematic review of intrathecal infusion systems for long-term management of chronic non-cancer pain. Pain Physician 2009; 12:345-360/

24. Manchikanti L, Boswell MV, Singh V, Benyamin RM, Fellows B, Abdi S, Buenaventura RM, Conn A, Datta S, Derby R, Falco FJE, Erhart S, Diwan S, Hayek SM, Helm S, Parr AT, Schultz DM, Smith HS, Wolfer LR, Hirsch JA. Comprehensive evidence-based guidelines for interventional techniques in the management of chronic spinal pain. Pain Physician 2009; 12:699-802.

25. Albright AL, Barron WB, Fasick MP, Polinko $P$, Janosky J. Continuous intrathecal baclofen infusion for spasticity of cerebral origin. JAMA 1993; 270:2475-2477.

26. Flückiger $B$, Knecht $\mathrm{H}$, Grossmann $\mathrm{S}$, Felleiter P. Device-related complications of long-term intrathecal drug therapy via implanted pumps. Spinal Cord 2008; 46:639-643.

27. Coffey RJ, Edgar TS, Francisco GE, Graziani V, Meythaler JM, Ridgely PM, Sadiq SA, Turner MS. Abrupt withdrawal from intrathecal baclofen: Recognition and management of a potentially life-threatening syndrome. Arch Phys Med Rehabil 2002; 83:735-741.

28. Green LB, Nelson VS. Death after acute withdrawal of intrathecal baclofen: Case report and literature review. Arch Phys Med Rehabil 1999; 80:1600-1604.

29. Steinbok P., O'Donnell M. Baclofen infusion for spastic cerebral palsy. Clin Neurosurg 2000; 47:440-57.

30. Penn RD, Savoy SM, Corcos D, Latash M, Gottlieb G, Parke B, Kroin JS. Intrathecal baclofen for severe spinal spasticity. N Engl J Med 1989; 320:1517-1521.

31. Grabb P, Guin-Renfroe S, Meythaler JM. Midthoracic catheter tip placement for intrathecal baclofen administration children with quadriparetic spasticity. Neurosurgery 1999; 45:833-840. 
